\title{
Durée étendue et visibilité de l'infime. À propos de films et de personnages de Lav Diaz
}

Extended Duration and Lowly Lives' Visibility. On Lav Diaz's Films and

Characters

Lucia Ramos Monteiro

\section{CpenEdition}

Journals

Édition électronique

URL : http://journals.openedition.org/recherchestravaux/1085

DOI : 10.4000/recherchestravaux.1085

ISSN : 1969-6434

Éditeur

UGA Éditions/Université Grenoble Alpes

Édition imprimée

ISBN : 978-2-37747-065-5

ISSN : 0151-1874

\section{Référence électronique}

Lucia Ramos Monteiro, «Durée étendue et visibilité de l'infime. À propos de films et de personnages de Lav Diaz », Recherches \& Travaux [En ligne], 93 | 2018, mis en ligne le 26 octobre 2018, consulté le 08 septembre 2020. URL : http://journals.openedition.org/recherchestravaux/1085 ; DOI : https:// doi.org/10.4000/recherchestravaux.1085

Ce document a été généré automatiquement le 8 septembre 2020

(c) Recherches \& Travaux 


\title{
Durée étendue et visibilité de l'infime. À propos de films et de personnages de Lav Diaz
}

\author{
Extended Duration and Lowly Lives' Visibility. On Lav Diaz's Films and
}

Characters

Lucia Ramos Monteiro

"Au-delà du texte philosophique, il n'y a pas une
marge blanche, vierge, vide, mais un autre texte,
un tissu de différences de forces sans aucun
centre de référence présente ${ }^{1}$."

1 Florentina Hubaldo, CTE (2012) s'ouvre avec l'image d'une route déserte, entourée par l'abondante végétation de la forêt qu'elle traverse, sous un ciel nuageux. Pendant les premières secondes, rien ne se passe au centre du cadre, sombre et vide. Notre regard se dirige alors vers les bordures latérales, où les feuilles bougent, et vers le haut, où les nuages se déplacent et la lumière change. C'est seulement après plus d'une minute de plan fixe sans action que nous apercevons que quelque chose à l'arrière-plan avance en notre direction. Au cours de ce long plan fixe, nous identifierons un homme âgé portant une chemise à carreaux, une jeune femme aux cheveux longs et deux chèvres tirées par une corde. Ils ne parlent pas, on ne sait pas où ils vont ni d'où ils viennent. Ce plan inaugural, d'une durée de cinq minutes, se limite à les montrer, entrant dans le cadre par le fond de l'image, pour en sortir par la portion inférieure gauche.

Dans l'ensemble du film, d'une durée de six heures au total, les cinq premières minutes servent moins à offrir les éléments narratifs et dramatiques introductoires qu'à instaurer un régime d'attention peu commun aujourd'hui, mais qui s'avérera dominant au cours de ce film - comme d'ailleurs dans une bonne partie du cinéma de son réalisateur, le Philippin Lav Diaz.

3 En effet, il s'agit d'un moment de «calibrage » de l'attention. Habitué à la vitesse croissante du montage actuel, de plus en plus rythmé par une parole devenue presque 
omniprésente, aussi bien dans les longs-métrages de fiction de grand public que dans le champ du documentaire, le spectateur doit gérer son impatience devant un film qui tarde à expliciter sa structure narrative, proposant par ailleurs l'exercice d'un regard centrifuge, à contresens des conventions de centralité et de perspective ${ }^{2}$. Nous sommes d'emblée incités à observer non pas ce qui se passe sur le point de convergence des quatre triangles dessinés par la rencontre entre route, forêt et ciel - situé légèrement en dessous et à droite du centre géométrique du cadre -, mais dans les marges de l'image.

4 Le regard vers des événements produits dans les "à-côtés " de l'image n'est pas quelque chose de nouveau dans l'histoire du cinéma - nombre de commentateurs du cinéma des frères Lumière ont pris note du frémissement des feuilles qui servaient de décor pour Le Repas de bébé (1895), dont «la mise en scène (...) est susceptible de décentrer l'attention portée à l'anecdote au profit de la dynamique interne au plan ${ }^{3}$ ». Indépendamment de l'inscription ou de l'action des personnages, les fonds cinématographiques de Diaz sont animés par des mouvements qui leur sont propres : ils sont visibles et audibles, ils agissent et se font remarquer, comme pour démarquer une ancienneté par rapport à toute intrigue. Chez Diaz il y a également une mise en rapport d'actions de rythmes différents, et le contraste est remarquable entre, d'un côté, l'agitation constante des feuilles, des nuages et des gouttes d'eau, et, de l'autre, la lenteur de la marche des personnages. Néanmoins, le regard décentré est dû surtout à la longue durée du plan et à la mise en scène qui, d'entrée de jeu, vide systématiquement l'espace connu comme "premier plan", le centre habituel de l'image cinématographique, pour ne le peupler que dans un deuxième temps, lorsque notre regard a déjà été habitué à une ballade centrifuge plutôt rare dans l'expérience du spectateur de cinéma.

6 Les lignes de force établies à l'ouverture de Florentina Hubaldo, CTE nous invitent même à aller au-delà des limites de l'image, prolongeant le regard vers ce qui se situe hors de l'écran - un hors-cadre qui est aussi hors-film. Cette configuration n'est pas, bien entendu, l'apanage de Florentina Hubaldo, CTE, ni même du cinéma de Lav Diaz. Tiago de Luca la situe dans le contexte d'un slow cinema particulièrement propice au visionnement en salle pour que «le contrat spectatoriel soit entièrement rempli ${ }^{4}$ ». Dans son analyse, De Luca observe les modalités spectatorielles engagées par ce style cinématographique fondé sur l'immobilité, le silence et la durée, un style qui serait impropre pour les petits écrans domestiques et pour le spectateur distrait de nos jours, et, en même temps, de plus en plus présent dans les galeries d'art. À partir de son étude de Goodbye, Dragon Inn (Tsai Ming-liang, 2003), Fantasma (Lisandro Alonso, 2006) et Shirin (Abbas Kiarostami, 2008), l'auteur affirme que, «par le biais d'une modalité d'adresse contemplative, le slow cinéma provoque une conscience accrue des conditions de visionnement ${ }^{5}$ ». La même remarque peut être faite à propos du cinéma de Lav Diaz en général et de Florentina Hubaldo, CTE en particulier, même si le visionnement domestique sert à apaiser l'angoisse du chercheur devant un film qui ne cesse de le fuir ${ }^{6}$.

7 Il est par ailleurs possible de voir des rapports entre le cinéma de Lav Diaz et le «durational cinema» proposé par Michael Walsh, dans la lignée des expériences de Andy Warhol (Empire, 1964) et Michael Snow (Wavelenght, 1967), qui suppriment l'intérêt du drame et de l'incident et placent le temps comme élément formel central'. Il s'agit, en effet, d'un cinéma qui donne à voir de manière spécialement intense le 
passage du temps. Dans le cas spécifique des productions du XXI ${ }^{e}$ siècle analysées par Walsh - comme dans celui de Diaz -, il s'agit d'une production réalisée et diffusée digitalement, alors que le support numérique avait été considéré comme moins capable d'offrir le sentiment de durée ${ }^{8}$.

Donnée récurrente du cinéma de Lav Diaz, dont les films dépassent fréquemment les deux heures conseillées par Hitchcock parce que compatibles avec la capacité de la vessie humaine, la longue durée non seulement nous permet de voir le passage du temps, notamment lors d'actions lentes qui nous sont rendues en temps réel, mais aussi favorise une sorte d'empathie avec les longs et douloureux processus historiques des Philippines. Ici il s'agira surtout de comprendre son impact sur l'expérience du spectateur en ce qui concerne spécifiquement la visibilité de l'infime. Selon notre hypothèse, la durée étendue des films de Diaz s'offrirait en tant que condition privilégiée pour l'observation de micro-événements - telles les feuilles qui bougent particulièrement nécessaire pour les récits de Lav Diaz, consacrés à des personnages à la marge des histoires officielles comme de la littérature.

Pour ce faire, nous nous concentrerons sur l'analyse de Florentina Hubaldo, CTE. Dans un premier temps, nous traiterons de la place faite aux marges et aux marginaux dans la cinématographie de Diaz à partir de ce film. Dans un deuxième temps, nous montrerons que, par sa longe durée, mais aussi en raison de la composition des cadres et de la structure narrative, marquée par le retour de lieux, cadres et monologues, avec des répétitions qui se conjuguent différemment à chaque fois, comme dans une spirale, Florentina Hubaldo stimule et sollicite la mémoire du spectateur soumis à une expérience proche de celle vécue par la protagoniste ${ }^{9}$. Afin d'offrir un éclairage sur ce jeu de miroirs délicatement construit entre trois niveaux de mémoire (mémoire du personnage/mémoire nationale/mémoire du spectateur) qui ne cessent de s'alimenter mutuellement, ce texte interroge la place consacrée au spectateur, et tente de comprendre la gestion d'un équilibre précaire entre attention, distraction, mémoire et oubli.

\section{La visibilité des marges}

10 Travailler le concept de limite revient à travailler à la limite du concept, nous dit Jacques Derrida, qui défend l'impossibilité de penser aux marges sans penser à ce qu'elles encerclent. Il ouvre son Marges de la philosophie avec un essai sur le tympan, charnière oblique entre l'intérieur et l'extérieur du corps. Le philosophe peuple alors les marges de sa page d'un extrait de "Perséphone », publié par Michel Leiris dans Biffures ${ }^{10}$. À côté de l'écriture derridienne, les ornements que Leiris décrit (la guirlande, l'enroulement, l'arabesque, les circonvolutions cérébrales, «la feuille d'acanthe qu'on copie au lycée quand on apprend à manier tant bien que mal le fusain », etc. ${ }^{11}$ ) offrent une visibilité nouvelle aux espaces qui entourent le texte "principal», réduisant la centralité de la page.

11 S'il y a des marges du cinéma, il y a encore un cinéma, le cinéma ? Il s'agit d'interroger, en paraphrasant Derrida, l'existence même du cinéma à une époque où les formats de prise de vue et de projection se voient transformés radicalement, et où l'expérience du spectateur semble avoir retrouvé une condition proche de celle du cinéma des premiers temps, avant que les conventions ne s'établissent. Chaque film, chaque situation de visionnement, invente un nouveau spectateur, et l'expérience de la salle devient, petit à 
petit, elle aussi marginale. À nouveau, un art parmi d'autres, regardé par des spectateurs assis, mais aussi allongés ou en mouvement, le cinéma contemporain redevient marginal, rareté sans aura. Ici, la proposition de l'impureté énoncée par André Bazin se révèle effectivement visionnaire :

Comme ces fleuves qui ont définitivement creusé leur lit et qui n'ont plus la force de mener leurs eaux à la mer sans arracher un grain de sable à leurs rives, le cinéma approche de son profil d'équilibre. [...] En attendant que la couleur ou le relief rendent provisoirement la primauté à la forme et créent un nouveau cycle d'érosion esthétique, le cinéma ne peut plus rien conquérir en surface. Il lui reste à irriguer ses rives, à s'insinuer entre les arts dans lesquels il a si rapidement creusé ses gorges, à les investir insidieusement, à s'infiltrer dans le sous-sol pour forer des galeries invisibles. Le temps viendra peut-être des résurgences, c'est-à-dire d'un cinéma à nouveau indépendant du roman et du théâtre. [...] En attendant que la dialectique de l'histoire de l'art lui restitue cette souhaitable et hypothétique autonomie, le cinéma assimile le formidable capital de sujets élaborés, amassés autour de lui par les arts riverains au cours des siècles. Il se l'approprie parce qu'il en a besoin, et que nous éprouvons le désir de les retrouver à travers lui ${ }^{12}$.

Lav Diaz puise dans les « arts riverains du cinéma » de différentes manières. D'une part, ses films commencent à être vus dans des espaces dédiés à l'art contemporain ${ }^{13}$. D'autre part, ses films s'ouvrent pleinement à la littérature ${ }^{14}$, à la musique ${ }^{15}$, au théâtre et aux arts visuels ${ }^{16}$.

La porosité d'un art cinématographique qui serait par définition marginal se double, chez le réalisateur, d'une façon étonnante de rendre visibles les marges - culturelles, artistiques, naturelles. Par l'agencement rhizomique de ses personnages, par la composition de ses plans et par la manière dont ses films s'insèrent dans une habitude et une culture cinéphilique, il semble affirmer que les puissances des lisières $-\mathrm{du}$ cinéma, du cinéma philippin - se situeraient justement dans leur impureté, leur porosité, leur dynamisme propre aux interstices : la dissolution des mythes nationaux et des frontières par les expériences artistiques ${ }^{17}$.

Notre centre d'intérêt ici réside dans la visibilité des êtres marginaux qui peuplent la filmographique de Diaz, de Batang West Side (2001), consacré à l'enquête menée par un inspecteur philippin établi aux États-Unis sur les morts mystérieuses de jeunes compatriotes exilés, liées au trafique d'une drogue nouvelle, à The Woman Who Left (2016), sur une femme qui sort de prison après y avoir passé trente ans enfermée injustement. Le réalisateur philippin renouvelle constamment son intérêt pour des "vies singulières devenues poèmes ", des existences situées entre l'anecdote véridique et la légende qui se heurtent au pouvoir. Dans leurs malheurs singuliers, de tels personnages, comme les infâmes collectionnés par Foucault, semblaient destinés à l'oubli avant qu'un cinéaste ne décide de leur conférer une forme filmique qui combine, elle aussi, réalité et fiction.

Dans le parcours proposé par la filmographie de Diaz, la figure d'Andrés Bonifacio est peut-être celle qui condense de la manière la plus prononcée les contradictions du texte foucaldien: l'un des fondateurs du Katipunan, mouvement ayant lutté pour l'indépendance des Philippines contre la couronne espagnole, Bonifacio (1863-1897) a été assassiné dans un complot, son corps n'a jamais été retrouvé et sa trajectoire n'a qu'une place obscure dans l'histoire philippine. Lav Diaz consacre à Andrés Bonifacio une place centrale dans Berceuse pour un sombre mystère (2016), film de huit heures dans lequel ce « héros noir » est pourtant physiquement absent - nous ne voyons que les 
gestes réitérés de sa femme, Gregoria de Jesus (Hazel Orencio), qui le cherche sans succès et prononce sans cesse son nom, comme des cris contre l'oubli.

L'existence de Bonifacio dans le film de 2016 fonctionne en quelque sorte comme un contrepoint à celle du personnage-titre de Florentina Hubaldo, CTE, réalisé par Diaz quatre ans auparavant. Il s'agit d'un personnage fictif, mais construit à partir d'une série d'éléments réels. Au début du film, un homme offre au père de Florentina 3000 pesos pour acheter ses deux chèvres, et 500 pesos pour sa fille. Dans des entretiens, Diaz affirme s'être inspiré d'une histoire réelle, racontée par un ami : appauvris, certains agriculteurs se voyaient obligés de vendre leurs filles pour acheter de quoi manger ${ }^{18}$.

En effet, Florentina Hubaldo, CTE dresse le portrait d'une femme constamment soumise aux violences de son père. Ce dernier humilie sa fille, la bat, la prostitue. Aidée par son grand-père, elle réussit à s'échapper, fuit, court, rêve, mais elle est sans cesse rattrapée et replongée dans sa routine de malheurs. Pendant six heures, nous assistons à la triste dynamique de cette vie, ainsi qu'aux nombreux efforts de Florentina pour se souvenir de son histoire, mais elle est à chaque fois confrontée à des trous de mémoire ainsi qu'à des souvenirs involontaires. Interprété avec brio par Hazel Orencio, actrice centrale dans la filmographie de Diaz, le personnage-titre tente à plusieurs reprises de raconter son histoire, mais elle perd le fil, oublie des choses, mélange tout. " J'ai mal à la tête. Je n'arrive pas à me souvenir de tout. Il y a plusieurs choses que j'ai déjà oubliées ", ditelle.

Les troubles amnésiques dont souffre Florentina (comme les abus auxquels elle est soumise) font écho à l'histoire nationale philippine ${ }^{19}$. Au niveau diégétique, la maladie neurodégénérative connue sous le sigle « $\mathrm{CTE}$ » dont Florentina est atteinte est due aux attaques de son père; au niveau allégorique, l'expérience de la colonisation espagnole suivie par l'occupation américaine et par la dictature de Marcos, engendrent également les éléments d'une mémoire post-traumatique. Si d'un côté cet entrelacement entre destin individuel et histoire nationale pourrait inscrire le film dans la tradition des "récits de fondation ", d'un autre côté les choix formels particuliers opérés par Diaz confèrent à cette catégorie un sens nouveau, la transforment. L'espoir d'une rédemption future, qui marque les récits de fondation traditionnels, se retrouve évacué, remplacé par le surgissement de fantômes, par la menace de reproduction perpétuelle d'un passé de souffrances ${ }^{20}$.

19 La vie infime de cette femme prisonnière de sa mémoire et de ses oublis fonctionne comme allégorie du destin national philippin, ou, selon les mots de Nadin Mai, comme " une métaphore du traumatisme collectif et de la souffrance nationale philippine, après des siècles de colonialisme suivis par des décennies de dictature sous le président Ferdinand Marcos, qui a soumis le pays à la loi martiale en septembre $1972^{21}$. »

Florentina Hubaldo, CTE se double d'une histoire parallèle, celle de Manoling (Noel Domingo) et Juan (Willy Fernandez), deux jeunes gens qui rendent visite à Hector (Joel Ferer), le frère du premier, à la recherche d'un trésor qui aurait été laissé par leur grand-père. Les deux histoires sont au fond liées mais se passent dans des temps différents : après la mort de Florentina, Hector cherche à rencontrer Loleng (Kristine Kintana), fille de Florentina, handicapée parce que sa mère a été maltraitée pendant la grossesse. 


\section{Attention et distraction}

21 À quelques différences près, la même composition décrite au début de ce texte se répète dans Florentina Hubaldo, CTE après une heure de film. La route du début est remplacée par un cours d'eau, et la durée du plan double : dix minutes. Par commodité d'écriture, ce plan sera appelé ici « plan du canal ». Comme au début, l'image peut être divisée en quatre parties : à gauche et à droite, frémissent des branches (non pas de bouleau, mais d'un arbuste de petite envergure) ; en haut, un ciel chargé de nuages ; en bas, de l'eau. La forêt a été remplacée par une plantation (de riz, probablement), et il n'y a plus de formes triangulaires, même approximatives. La ligne d'horizon est cette fois-ci visible, un peu inclinée, comme si l'opérateur (Lav Diaz lui-même) avait mal réglé son trépied. Une pluie fine agite l'eau stagnante du canal. Comme au début, nous voyons quelque chose qui avance vers le premier plan. Comme au début, au son diffus du vent, s'ajoute le chant lointain d'un coq. Petit à petit, nous identifions un homme : il porte une casquette. Impossible de dire dès le départ s'il se situe à droite du canal, à gauche ou, peut-être - et c'est le cas - s'il ne marche pas dans l'eau. Il tient quelque chose dans sa main droite. Un bâton? Un couteau? Il s'agit d'un nouveau début, qui marque l'introduction d'un nouveau personnage dans la trame: Hector, que nous regarderons creuser des trous dans le sol à la recherche d'un mystérieux trésor, et plus d'une fois les pieds dans l'eau comme lors de cette apparition initiale. Il s'approche de la caméra, coupe une branche de l'arbuste qui orne toute la portion gauche du cadre, s'assoit à la marge. La tête inclinée devant lui, il a le regard perdu vers l'eau. En off, nous entendons : « Pourquoi y a-t-il de la douleur? Pourquoi y a-t-il du chagrin? »

L'étrange temporalité du film produit sur le spectateur une réaction étonnante : le plan de dix minutes n'est pas perçu comme deux fois plus long que celui de cinq minutes; il est possible que l'impatience du public ne s'accentue pas, voire même diminue. Si l'exercice peut être perçu comme peu commode, surtout au début, au risque de provoquer l'ennui ou de précipiter le sommeil, ceux qui acceptent les règles de ce contrat inhabituel s'y habituent petit à petit, et ces deux plans presque "vides " d'action se voient particulièrement propices à un exercice actif de mémoire, de sorte que le premier « revient » à la vision du second. Quand le plan du canal survient, après une heure de film, chaque spectateur est déjà sensibilisé au rythme particulier de Florentina Hubaldo, CTE, a déjà développé ses propres stratégies de regard. La durée du plan est spécialement favorable à l'acte de mémoire, et le premier plan du film revient dans notre souvenir.

Entre mémoire et oubli, distraction et attention, le spectateur des films-fleuve de Lav Diaz navigue dans un équilibre instable, au sein duquel la bande sonore joue un rôle fondamental. Vers le milieu du film, après quelque 160 minutes, un plan silencieux de huit minutes répète une troisième fois la configuration $\mathrm{du}$ " plan de la route » et $\mathrm{du}$ "plan du canal». Il s'agit du "plan de la rue», complètement flou au début, qui commence de nuit, sous la pluie. La rue est filmée de face, laissant apparaître, sur sa gauche, des maisons, un camion garé avec les clignotants en warnings et des lampadaires dont la lumière se reflète sur l'asphalte humide. La portion droite du cadre est plus sombre, et c'est de là, au fond, que surgira Florentina, marchant d'un pas décidé en direction de la caméra. Elle s'arrête lorsque son corps est suffisamment proche pour être vu en plan américain et qu'il devient net. Elle affronte l'objectif, droit dans les yeux. Impossible de présupposer l'emplacement d'un quelconque personnage là 
où la caméra se situe : c'est dans notre direction que le personnage regarde, et c'est à nous qu'elle tend le bras, se maintenant dans cette position malgré la pluie qui s'intensifie.

24 Le regard centrifuge est stimulé par les vibrations lumineuses, par la pluie qui tombe ainsi que par le passage de quelques véhicules, le spectateur est par ailleurs incité à la remémoration des plans précédents aux configurations proches. Le "plan de la rue » interpelle le spectateur également par la performance de Hazel Orencio, qui maintient ce peu commode regard vers la caméra pendant de longues minutes, malgré les perturbations de la pluie et le passage d'autres voitures. Nous sommes interpellés aussi par le silence de la bande sonore à ce moment-là - il s'agit d'une véritable absence de son: aucune ambiance, aucune voix, aucun bruit - et par la façon dont les gestes du personnage s'adressent à nous. Tente-t-elle de s'échapper de l'espace filmique, ou bien s'efforce-t-elle de nous faire sortir de notre position de spectateur ${ }^{22}$ ? Se positionner devant la caméra, la regarder, tendre le bras dans sa direction. Non seulement les règles de la "suture ${ }^{23}$ " se trouvent brisées, mais toute possibilité de passivité ou de léthargie du spectateur est également compromise.

\section{Considérations finales}

Jacques Aumont établit trois catégories pour marquer le changement de format entre plan long et plan séquence. Il identifie d'abord le "plan statique », qui est "organisé dans la profondeur, où plusieurs actions se déroulent en même temps ». Vient ensuite un type de plan "très mouvementé, où l'on désire avant tout explorer un espace ", au sein duquel «le spectateur n'est pas particulièrement "libre" ", mais où " on le prend par la main, [où] on lui fait voir l'un après l'autre des éléments de l'action». En troisième lieu, Aumont place le " plan long, simplement tenu très longtemps, où c'est le passage du temps qui devient le facteur le plus important». Si les trois plans sur lesquels je me suis arrêtée ici sont "statiques » et "organisés en profondeur", c'est cette troisième catégorie qui décrit de manière plus pertinente le cinéma de Lav Diaz ${ }^{24}$. Néanmoins, le montage en spirale mis en place dans Florentina Hubaldo, CTE réconcilie deux aspects vus par Aumont comme contradictoires. Le fait de pousser «à sa limite une certaine logique du plan " pour mettre l'accent sur "sa valeur d'unité autonome " a pour conséquence la diminution de «sa fonction d'élément d'un ensemble ». Il est vrai que chez Diaz les plans sont des unités pleines de sens, explorées dans leurs limites, comme l'atteste le plan final, de plus de vingt minutes, pendant lequel Florentina Hubaldo se confie à la caméra jusqu'à s'évanouir, l'épuisement du personnage laissant entrevoir l'état de fatigue de l'actrice. Et pourtant, c'est l'accumulation des plans qui éduque le regard du spectateur - et l'expérience de visionner des plans séparés, même les plus longs, est loin d'être aussi radicale que la séance intégrale. L'insistance avec laquelle les configurations se répètent et se modifient agit sur la mémoire du spectateur, et les plans du début reviennent en tant qu'éléments d'un ensemble plus large qui acquiert, dans la longueur, de nouvelles couches de sens.

Chez Lav Diaz, il s'agit d'un recours nécessaire pour que le spectateur puisse comprendre, de façon paradoxale, l'ampleur des vies infimes : elles ne sont pas entrevues comme dans l'éclair benjaminien ${ }^{25}$, mais se montrent dans la durée de leur 
souffrance, dans la persistance de leur affrontement aux autorités, dans la lutte entre mémoire et oubli.

Dans la suite de ce que dit Tiago de Luca à propos de la conscience accrue du spectateur $\mathrm{du}$ « slow cinema » par rapport aux conditions de visionnement, voir les films de Diaz, et Florentina Hubaldo, CTE en particulier, revient alors à réfléchir sur la posture spectatorielle contemporaine, et sur l'état actuel - marginal ? - du cinéma. Les séances collectives de Diaz réitèrent la sensation d'un hic et nunc partagé, d'événements vécus collectivement, et une communauté de spectateurs se forme dans le cadre de ces expériences particulières où l'on reconnaît son voisin de siège. Cette communauté de spectateurs dédouble en quelque sorte l'idée de communauté imaginaire, concept forgé par l'historien Benedict Anderson à partir de ses études sur l'indépendance philippine et la construction du sentiment national. Anderson se base notamment sur les pages de l'écrivain indépendantiste philippin José Rizal (1861-1896), source d'inspiration pour Berceuse pour un sombre mystère (2016) de Diaz. Ce qu'écrit Anderson à propos de la littérature de Rizal pourrait être appliqué au cinéma de Lav Diaz : « la fiction s'infiltre silencieusement dans la réalité, et crée cette admirable confiance de la communauté dans l'anonymat qui constitue la marque des nations modernes ${ }^{26} »$.

\section{NOTES}

1. Jacques Derrida, Marges de la philosophie, Paris, Minuit, 1972, p. XIX.

2. Des recherches sur la durée moyenne des plans, ou average shot length (ASL), ainsi que sur la quantité moyenne de plans d'un film indiquent une accélération du montage dans la production contemporaine - avec, dans certains titres, une durée moyenne inférieure à quatre secondes par plan, tandis que dans Florentina Hubaldo, CTE, elle est de plus de deux minutes, et arrive à trois minutes dans d'autres films de Diaz. Cf. Nadin Mai, The aesthetics of absence and duration in the post-trauma cinema of Lav Diaz, University of Stirling, 2015 (thèse de doctorat), p. 40-41.

3. Robert Bonamy, Le fond cinématographique, Paris, L'Harmattan, 2013, p. 20.

4. Tiago de Luca, «Slow Time, Visible Cinema: Duration, Experience, and Spectatorship ", Cinema Journal, vol. 56, $\mathrm{n}^{\circ} 1,2016$, p. 23.

5. Tiago de Luca, «Slow Time, Visible Cinema: Duration, Experience, and Spectatorship ", Cinema Journal, vol. 56, n ${ }^{\circ}$, 2016, p. 23-25.

6. À propos de la longue durée au cinéma et les des difficultés qu'elle engage pour le travail d'analyse filmique, cf. Lúcia Ramos Monteiro, « $\mathrm{O}$ cinema existe e resiste. Longa duração, análise fílmica e espectatorialidade nos filmes de Lav Diaz », Aniki, vol. 4, no 2, 2017, p. 434-455, ainsi que les remarques finales de Raymond Bellour à propos d'installations-vidéo de longue durée dans "Trente-cinq ans après, le "texte" à nouveau introuvable?", dans Images contemporaines, L. Vancheri (ed.), Lyon, Aléas, 2009, p. 17-33.

7. Michael Walsh, «The First Durational Cinema and the Real of Time », dans Tiago de Luca and Nuno Barradas Jorge (orgs.), Slow Cinema, Edinburgh, Edinburgh University Press, 2016. 
8. Voir notamment les observations de Babette Mangolte dans « Afterword: A Matter of Time ", dans Richard Allen and Malcolm Turvey (dir.), Camera Obscura, Camera Lucida: Essays in Honor of Annette Michelson, Amsterdam, Amsterdam University Press, 2002; ainsi que celles de D. N. Rodowick, dans The Virtual Life of Film, Cambridge, MA, Harvard University Press, 2007. Jihoon Kim les reprend dans « Expressing Duration with Digital Micromanipulations: Digital Experimental Documentaries of James Benning, Sharon Lockhart, and Thom Andersen », Cinema Journal, vol. 57, n 3, 2018, p. 101-125.

9. Pour Nadin Mai, Florentina se situe dans un « récit cyclique, conséquence de l'événement traumatique, qui l'enferme dans son passé et dans une lutte pour se rappeler même du nom de sa mère » (The aesthetics of absence and duration in the post-trauma cinema of Lav Diaz, thèse de doctorat, Université de Stirling, 2015, p. 231).

10. Jacques Derrida, "Tympan", dans Jacques Derrida, Marges de la philosophie, Paris, Minuit, 1972, p. I-XXV ; Michel Leiris, «Perséphone », dans Michel Leiris, La Règle du Jeu I. Biffures. Paris, Gallimard, 1968.

11. M. Leiris, op. cit., p. 85-86; repris par Derrida dans « Tympan », p. I-III.

12. André Bazin, «Pour un cinéma impur. Défense de l'adaptation» (1951), Qu'est-ce que le cinéma ?, Paris, Cerf, 2010, p. 105-106.

13. Notamment lors de la rétrospective londonienne de 2017, organisée par May Adadol Ingawanij, Michael Mazière, George Clark et Julian Ross, qui s'est presque entièrement déroulée à la galerie London Gallery West.

14. Parmi les exemples, l'on peut citer Berceuse pour un sombre mystère inspiré de l'œuvre de José Rizal ou The Whoman who left inspiré de Tolstoï.

15. Voir notamment la place donnée au genre chansonnier « kundiman » dans Berceuse...

16. Plusieurs moments de Florentina Hubaldo, CTE et The Woman Who Left témoignent d'une logique propre à la performance.

17. Ces réflexions n'auraient pas été possibles sans le colloque Puissances esthétiques des lisières culturelles, organisé par Nedjma Moussaoui et Dario Marchiori à l'Université Lumière - Lyon 2 en novembre 2016, où elles ont été formulées pour le première fois.

18. Cf. Nadin Mai, op. cit., p. 234.

19. La dialectique entre mémoire et oubli constitue une véritable obsession de Lav Diaz, de Batang West Side (2001), où le protagoniste (un jeune Philippin vivant dans le New Jersey) reçoit de son grand-père des livres et des leçons d'histoire, à A Lullaby to the Sorrowful Mystery (2016), qui met en évidence un passage trouble de l'histoire de l'indépendance du pays.

20. Pour une catégorisation précise des récits de fondation, surtout en littérature, cf. l'étude de Doris Sommer, Foundational Fictions: The National Romances of Latin America, Berkeley, University of California Press, 1993.

21. Cf. Nadin Mai, op. cit., p. 235.

22. Pour Nadin Mai, la mise en scène de Florentina Hubaldo, CTE évoque l'expérience concentrationnaire (op. cit.).

23. Je fais référence au texte de Jean-Pierre Oudart, publié dans les Cahiers du Cinéma (no 211 et no 213, avril/mai 1969), qui transpose dans le cinéma le concept lacanien de séparation entre sujet et discours. La "suture " cinématographique fait écho à l'idée de "quatrième mur", formulée originalement par Denis Diderot pour désigner le mur invisible qui assure la séparation entre la scène théâtrale et les spectateurs. Pour Oudart, grâce aux règles de la "suture ", présidant notamment le jeu du champ/contrechamp, les coupes du montage cinématographique ne sont pas perçues par le spectateur, qui réussit à recomposer mentalement un espace filmique unifié et reste ainsi attaché au discours filmique. Je ne reviens pas ici sur les nombreuses critiques faites à cette proposition, notamment celles de David Bordwell (Narration in the fiction 
film, Madison, University of Wisconsin Press, 1985), pour qui Oudart a négligé la diversité des styles cinématographiques.

24. Jacques Aumont, Le Montage " la seule invention du cinéma », Paris, Vrin, 2015, p. 59-61.

25. Walter Benjamin, «Thèses sur le concept d'histoire » (1940), thèse V, dans Écrits français, Paris, Gallimard, 1991, p. 435.

26. Benedict Anderson, Les bannières de la révolte, trad. Émilie L'Hôte, Paris, La Découverte, 2009, p. 69.

\section{RÉSUMÉS}

Dans le cadre de la pensée sur la longue durée et la lenteur dans le cinéma contemporain, ce texte offre une analyse de Florentina Hubaldo, CTE (2012), film de six heures réalisé par le réalisateur philippin Lav Diaz. Nous nous concentrerons sur quelques-uns de ses plans longs et très longs pour dessiner une articulation entre leur durée étendue, et la visibilité conférée à des êtres infimes et marginaux, associés de manière allégorique à l'histoire nationale.

In the context of a large discussion on long duration and slowness in contemporary cinema, this article offers an analysis of Florentina Hubaldo, CTE (2012), a six-hour film by Lav Diaz. We focus on certain long shots in order to propose a relation between extended duration and the lowly lives' visibility, associated in an allegorical way to the Philippines national history.

\section{AUTEUR}

\section{LUCIA RAMOS MONTEIRO}

Lucia Ramos Monteiro est chercheuse associée à l'École de communication et arts de l'Université de Sao Paulo, où elle enseigne, avec un financement Fapesp. Titulaire d'un doctorat en Études cinématographiques réalisé en co-tutelle internationale (Université Sorbonne Nouvelle Paris 3 et Université de Sao Paulo), elle travaille par ailleurs en tant que programmatrice, en organisant des cycles de films au Brésil, en Colombie, en France et en Suisse, en plus d'être critique de cinéma et arts visuels. Elle a dirigé les ouvrages Oui, c'est du cinéma (Campanotto, 2009), sur cinéma et art contemporain ; Palmanova (Form[e]s, 2016), autour de l'œuvre de Victor Burgin ; et Cinema e história (Sulinas, 2017), sur l'articulation entre recherche historiographique et analyse esthétique du cinéma. 